\title{
MiRNA-15a Mediates Cell Cycle Arrest and Potentiates Apoptosis in Breast Cancer Cells by Targeting Synuclein- $\gamma$
}

\author{
Ping Li1, ${ }^{1,2}$ Xiao-Bing Xie ${ }^{2}$, Qian Chen ${ }^{3}$, Guo-Lian Pang ${ }^{4}$, Wan Luo ${ }^{1}$, Jian-Cheng \\ Tu$^{1}$, Fang Zheng ${ }^{1}$, Song-Mei Liu ${ }^{1}$, Lu Han ${ }^{1}$, Jian-Kun Zhang ${ }^{4}$, Xian-Yong Luo ${ }^{4}$, \\ Xin Zhou ${ }^{1 *}$
}

\begin{abstract}
Background: Recent studies have indicated that microRNA-15a (miR-15a) is dysregulated in breast cancer (BC). We aimed to evaluate the expression of miR-15a in BC tissues and corresponding para-carcinoma tissues. We also focused on effects of miR-15a on cellular behavior of MDA-MB-231 and expression of its target gene synuclein- $\gamma$ (SNCG). Materials and Methods: The expression levels of miR-15a were analysed in BC formalin fixed paraffin embedded (FFPE) tissues by microarray and quantitative real-time PCR. CCK-8 assays, cell cycle and apoptosis assays were used to explore the potential functions of miR-15a in MDA-MB-231 human BC cells. A luciferase reporter assay confirmed direct targets. Results: Downregulation of miR-15a was detected in most primary BCs. Ectopic expression of miR-15a promoted proliferation and suppressed apoptosis in vivo. Further studies indicated that miR-15a may directly interact with the 3'-untranslated region (3'-UTR) of SNCG mRNA, downregulating its mRNA and protein expression levels. SNCG expression was negatively correlated with miR-15a expression. Conclusions: MiR-15a has a critical role in mediating cell cycle arrest and promoting cell apoptosis of BC, probably by directly targeting SNCG. Thus, it may be involved in development and progression of BC.
\end{abstract}

Keywords: microRNA-15a - breast cancer - SNCG - cell cycle - apoptosis

Asian Pac J Cancer Prev, 15 (16), 6949-6954

\section{Introduction}

$\mathrm{BC}$ is the most common cancer in women worldwide, with about 1.38 million new cases and 458, 000 deaths each year (Ferlay et al., 2010). The majority of BC morbidity and mortality results from incurable metastatic disease that is highly resistant to conventional therapies. To reduce $\mathrm{BC}$ mortality it is therefore essential to further elucidate the molecular mechanisms of $\mathrm{BC}$ metastasis, and develop novel therapeutic approaches. Although decades of metastasis research have provided considerable insight into the multistep metastatic process, there are still significant gaps in our knowledge.

Recently, it has been recognized that microRNAs (miRNAs) affect various steps in BC metastasis including migration, invasion and angiogenesis and could represent potential therapeutic targets (Harquail et al., 2012; Wang et al., 2012). MiRNAs are short non-coding RNAs that target messenger RNAs (mRNAs) to function as key posttranscriptional regulators of gene expression (Ambros, 2001). By binding to the 3'-UTR of their target mRNAs, miRNAs inhibit translation and/or promote mRNA degradation (Valencia-Sanchez et al., 2006). Consequently, they can act either as oncogenes or as tumor suppressors depending on their target mRNAs (Garzon et al., 2009; Shenouda et al., 2009). MiRNAs play important roles in various biological processes, including development, differentiation, apoptosis, and cell proliferation (Bartel, 2004). However, very few studies have reported miRNA deregulation in BC (Yamanaka et al., 2009; Ng et al., 2011; Paik et al., 2011). Thus, the functional roles of miRNAs in $\mathrm{BC}$ are still not fully understood.

To determine which miRNA plays a role in $\mathrm{BC}$, we conducted miRNA microarray analysis, and found that miR-15a was downregulated. We further found that miR-15a led to decreased expression of SNCG, thereby resulting in inhibition of cell cycle progression and promotion of cell apoptosis. Jointly, these data implicate that downregulation of miR-15a functions critically in blocking cell cycle arrest and inhibiting cell apoptosis of $\mathrm{BC}$, likely by directly targeting SNCG.

\section{Materials and Methods}

Breast cancer FFPE tissue and quality control

This study was approved by the Ethics Committee

${ }^{1}$ Center for Gene Diagnosis, Zhongnan Hospital of Wuhan University, Wuhan, ${ }^{2}$ Medical Laboratory Center, First Affiliated Hospital, Hunan University of Chinese Medicine, Changsha, ${ }^{4}$ Department of Pathology, First People Hospital of Qujing, Qujing, China, ${ }^{3}$ Department of Molecular Biology and Genetics, Johns Hopkins University School of Medicine, Baltimore, USA *For correspondence: zhouxinwhu@163.com 
Ping Li et al

of Zhongnan Hospital of Wuhan University. Informed consent was obtained from all subjects. 172 FFPE breast tissues (including $106 \mathrm{BC}$ tissues, and 66 paracarcinoma tissues paired to $\mathrm{BC}$ ) were collected from the tissue repository in the First People's Hospital of Qujing in Yunan province from Jan 2011 to May 2013. None of the patients received preoperative treatment such as radiotherapy or chemotherapy. All of the FFPE tissues were evaluated histologically by two certified pathologists, and the area composition of invasive ductal carcinoma was confirmed in more than $80 \%$ of malignant epithelial cells under the microscope. Para-carcinoma tissues were controlled within $2 \mathrm{~cm}$ from cancer tissue and disallowed to exist cancer cells. Each FFPE tissue block was cut into 4 pieces of $20 \mu \mathrm{m}$ thick sections and collected in a $2 \mathrm{ml}$ RNA enzyme-free tube.

RNA extraction from FFPE tissue and quantitative realtime PCR ( $q R T-P C R)$

Total RNA was extracted from FFPE breast tissue by using miRNeasy FFPE Kit (Qiagen, Germany) according to the manufacturer's instructions. The concentration and purity of all RNA samples were detected by a NanoDrop 2000 spectrophotometer (Thermo Scientific, Germany).

Expression of miR-15a was assayed using stem-loop RT followed by qRT-PCR analysis, as the document described (Chen et al., 2005). All reagents for stem-loop RT and qRT-PCR were obtained from Thermo Scientific (Germany). qRT-PCR was examined in triplicate and calculated using the $2^{-\Delta \mathrm{CT}}$ method (Lian et al., 2009), where $\Delta \mathrm{Ct}=\mathrm{Ct}_{\text {miR-15a }}-\mathrm{Ct}_{\mathrm{U} 6}$.

Expression of SNCG mRNA was examined in triplicate and calculated using the $2^{-\Delta \Delta \mathrm{CT}}$ method, where $\Delta \Delta \mathrm{Ct}=\Delta \mathrm{Ct}{ }_{\text {miR-15a group }}-\Delta \mathrm{Ct}{ }_{\mathrm{NC} \text { group, }} \Delta \mathrm{Ct}=\mathrm{Ct}_{\mathrm{SNCG}}-\mathrm{Ct}{ }_{\beta \text {-actin }}$. The primers used for stem-loop RT and qRT-PCR are listed in Table 1.

\section{MiRNA microarray assay and data analysis}

Microarray was executed in $6 \mathrm{BC}$ tissues and 6 Paracarcinoma tissues paired with BC. The Affymetrix miRNA 3.0 Technology platform was used, which contains 1733 mature human miRNAs. Total RNA was exerted gradually by poly (A) tailing, biotin labeling, hybridization, washing, staining and scanning with Affymetrix Scanner 3000 (Affymetrix) according to the manufacturer's guidelines.

Expression Console software (version 1.3.1, Affymetrix) was used to analyze array images to get raw data and then offered RMA normalization. Next, Genesrping software (version 12.5, Agilent Technologies)

Table 1. The primers used for stem-loop RT and qRTPCR

\begin{tabular}{ll}
\hline Primers & \multicolumn{1}{c}{ Sequences } \\
\hline U6-F & 5'-CTCGCTTCGGCAGCACA-3' \\
U6-R & 5'-AACGCTTCACGAATTTGCGT-3' \\
miR-15a-RT & 5'-GTCGTATCCAGTGCAGGGTCCGAGGTA \\
& TTCGCACTGGATACGACCACAAACC-3' \\
miR-15a-F & 5'-GCTAGCAGCACATAATGGTTTGTG-3' \\
miR-15a-R & 5'-GTGCAGGGTCCGAGGTATTC-3' \\
SNCG-F & 5'-GCATCAATATTTCATCGGCGTCA-3' \\
SNCG-R & 5'-TCTTCTCAGCTGCTTCCGTCA-3' \\
*F: Forward, R: Reverse, RT: Reverse Transcription
\end{tabular}

was used in the following data analysis. Probes that at least one group out of all samples have flags in "P" were chosen for further data analysis. Differentially expressed miRNAs were then identified through fold change and $\mathrm{P}$ value comparison. The stringent threshold set for up- and down-regulated genes was a fold change $\geq 2.0$ and $p \leq 0.05$. Unsupervised hierarchical clustering was performed to show the distinguishable miRNAs expression pattern among samples.

\section{MiRNA mimics and cell transfection}

MDA-MB-231 cells were cultured in Dulbecco's Modified Eagle Media (DMEM) (GIBICO BRL, GrandIsIand, NY) with $15 \%$ Fetal Bovine Serum (FBS), and were maintained at $37^{\circ} \mathrm{C}$ in the presence of $5 \%$ $\mathrm{CO}_{2}$. MiR-15a mimics and negative controls (NC) were synthesized by GenePharma (Shanghai, China). They were transfected respectively into MDA-MB-231 cells at 100 $\mathrm{nM}$ by Lipofectamine $\mathrm{TM}^{\mathrm{TM}} 2000$ (Invitrogen) according to the manufacturer's instruction.

\section{Cell cycle and cell apoptosis analysis}

Forty eight hours after transfection, cells were harvested and approximately $0.5 \times 10^{6}$ cells were suspended in $500 \mu \mathrm{l}$ of propidium iodide (PI) solution $[20 \mu \mathrm{g} / \mathrm{ml} \mathrm{PI}$, $50 \mu \mathrm{l} / \mathrm{ml}$ RNaseA, $0.02 \% \mathrm{NP} 40$ in PBS] at $4^{\circ} \mathrm{C}$ for $30 \mathrm{~min}$. DNA content analysis was performed by a FACS Calibur instrument (Becton-Dickinson, Mountain View, CA) and CellQuest software (Becton-Dickinson). Cells were synchronized at G1/S transition by serum deprivation for $12 \mathrm{~h}$ and $2 \mathrm{mM}$ of hydroxyurea (HU) present for 1618h. Apoptotic cells were detected by FITC Annexin V apoptosis detection kit (BD Pharmingen) according to the protocols recommended by the manufacturer.

\section{Cell viability analysis}

Cells were seeded in 96-well plate at $3 \times 10^{5}$ cells $/ \mathrm{ml}$ in a volume of $100 \mu \mathrm{l}$ per well and transfected with miR15a mimics and NC. CCK- 8 was used to measure the absorbance value of OD $450 \mathrm{~nm}$ with microplate reader (Bio-Tek) at 24h intervals for 5-7 days. Each experiment was repeated three times.

\section{Western blot detection of the effects of miR-15a on SNCG expression}

We used three computational methods (miRNAorg, Targetscan and PITA data base) to search for the common targets of miR-15a in human. They were drawn by venn diagram and Cytoscape Software.

MDA-MB-231 cells cultured normally were uniformly inoculated into 6-well culture plates at a concentration of $3 \times 10^{5}$ cells $/ \mathrm{ml}$ in a volume of $1,000 \mu \mathrm{l}$ per well. The transfection of the miR-15a mimics and NC was performed according to the manufacturer's instructions using Lipofectamine ${ }^{\mathrm{TM}}$ 2000. Forty-eight hours after transfection, western blotting was used to measure the SNCG protein expression levels in the cells.

Each well of the 6-well cell culture plates was lysed in $1 \mathrm{ml}$ of Radio-Immuno precipitation Assay (RIPA) lysis buffer $[150 \mathrm{mM} \mathrm{NaCl}, 1 \% \mathrm{NP} 40,0.5 \%$ sodium deoxycholate, $0.1 \%$ SDS, $50 \mathrm{mM}$ Tris ( $\mathrm{pH} 7.9), 10 \mathrm{mM}$ 

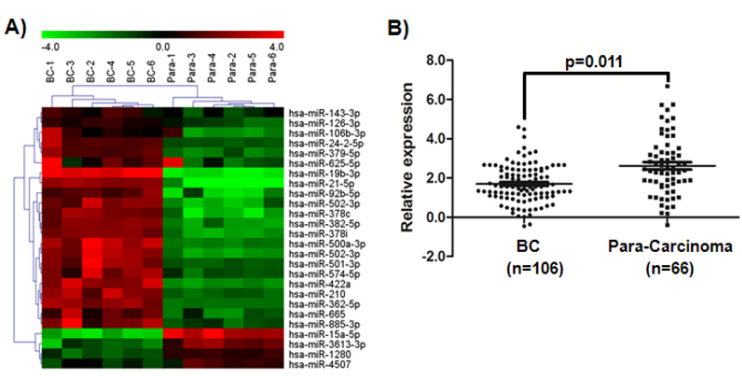

Figure 1. Differential Expressed miRNAs in Beast Cancer. A). Color gradation indicates the relative expression level of miRNAs from low expression (green) to high expression (red). B). The relative expression of miR-15a was down-regulated in $\mathrm{BC}$ tissues $(\mathrm{n}=106)$ compared with para-carcinoma tissues $(n=66)$ by method of miRNA specific stem-loop RT-PCR $(p<0.05)$

NaF, 10mM PMSF, and $1 \times$ protease inhibitors (Complete Protease Inhibitor Cocktail tablets, Roche)], and the cell lysates were transferred to 1.5 - $\mathrm{ml}$ centrifuge tubes. After $30 \mathrm{~min}$ of centrifugation at $16,000 \times \mathrm{g}$, the supernatant was collected, and the supernatant protein concentration was measured using the bicinchoninic acid (BCA) method. A 5\% stacking gel and 15\% separation gel was cast, and each lane was loaded with $50 \mu \mathrm{g}$ protein, followed by electrophoretic separation. Proteins were wet transferred to PVDF membrane (Bio-Rad, USA), and the membrane was blocked in TBST $(10 \mathrm{mM}$ Tris- $\mathrm{HCl}, \mathrm{pH}$ $7.5,150 \mathrm{mM} \mathrm{NaCl}$, and $0.1 \%$ Tween-20) containing 5\% non-fat milk at room temperature for $1 \mathrm{~h}$. Subsequently, a rabbit anti-human SNCG polyclonal antibody (1:500 dilution) (Abcam, USA) and a mouse anti-human $\beta$-actin monoclonal antibody (1:1000 dilution) (Abcam, USA) were added, followed by overnight incubation at $4^{\circ} \mathrm{C}$. The corresponding IRDye 800 -labeled secondary antibodies (1:2000 diluted in PBS) were added, followed by overnight incubation at $4^{\circ} \mathrm{C}$. After washing with TBST, the membrane was scanned using the Odyssey Infrared Imaging System (Rockland). The relative levels of SNCG were represented by a SNCG/ $\beta$-actin grayscale ratio, and the grayscale was analyzed using the Quantity One software (Bio-Rad, USA).

\section{SNCG- 3'UTR luciferase reporter assay}

The 3' UTR sequences of the SNCG gene were amplified from the genomic DNA of normal tissues and then sub-cloned directly downstream of the stop codon of Renilla luciferase of psiCHECK2. Primer sequences used to amplify this region were SNCG3'UTR-F: 5-CCCTCG AGGAGTGGGGGAGACTAGAGGG-3' and SNCG3'UTR-R: 5'-GCAGGAGTGGGCTCAAGTTGC GGCCGCTTTA-3'. Both wild-type and mutant 3' UTR sequences were confirmed by sequencing. For the luciferase screens, MDA-MB-231 cells were seeded in 96-well plates at a density of 5,000 cells per well. After 24 hours, MDA-MB-231 cells were cotransfected with 25 pmol of miR-15a mimics or NC, along with 500ng of psiCHECK2-3'UTR-SNCG construct or psiCHECK23'UTR-SNCG mutation using Lipofectamine ${ }^{\mathrm{TM}} 2000$ per well, according to the manufacturer's protocol. Cells were grown for 48 hours, Firefly and Renilla luciferase activities were quantified using the Dual-Luciferase Reporter Assay System (Promega), and Renilla luciferase activity was normalized to firefly luciferase activity. For each experiment, a control employing an empty vector was used; corrected luciferase values were averaged, arbitrarily set to a value of " 1 ," and served as a reference for comparison of fold-differences in experimental values.

\section{Statistical analysis}

Statistical differences and variances were determined by Student $t$ tests. Average data was presented as mean \pm standard deviation (SD). $p<0.05$ was considered to be statistically significant.

\section{Results}

MiRNAs profiling in BC FFPE tissues and qRT-PCR

To identify miRNA that may suppress BC proliferation via epigenetic mechanisms, we performed miRNA microarray analysis in 6 BC FFPE tissues and 6 paracarcinoma tissues. Among the 26 differentially expressed miRNAs, miR-15a, miR-3613, miR-1280 and miR-4507 were downregulated (Figure 1A). However, the roles of either individual or global miR-15a in cancer are still poorly reported. Thus, we focused our study on the miR$15 \mathrm{a}$.

To validate the miRNA expression changes on the miRNA microarray, we employed qRT-PCR to measure the levels of miR-15a in 106 BC FFPE tissues and 66 paracarcinoma tissues. Consistent with the results by miRNA microarray; qRT-PCR confirmed the downregulation of the miR-15a in BC FFPE tissues $(p<0.05)$ (Figure 1B).

MiR-15a induces apoptosis, causes the G1 cell cycle arrest, and reduces cell viability

Forty eight hours later, MDA-MB-231 cells were transfected with $100 \mathrm{nM}$ of miR-15a mimics or NC. Cells were stained with Annexin V-FITC and PI, and apoptosis was measured by flow cytometry. In these experiments, transfection of MDA-MB-231 cells with miR-15a mimics caused statistically significant changes in the incidence of apoptosis in comparison to the $\mathrm{NC}$ group $(p<0.05)$ (Figure 2A, 2B).

To confirm that the expression of miR-15a can cause G1 arrest, MDA-MB-231 cells transfected with miR-15a mimics were synchronized at the $\mathrm{G} 1 / \mathrm{S}$ transition by serum starvation and Hydroxyurea (HU). DNA content was examined from the time of HU release. The results showed that all cells transfected with miR-15a mimics began to arrest at G1 phase and inhibited the transfection from G1 phase to $S$ phase. $(p<0.05)$ (Figure 3A, 3B).

Using a CCK-8 assay, we found that the cell viability of the miR-15a mimics transfection group was lower than that of the $\mathrm{NC}(p<0.05)$, suggesting that over-expression of miR-15a reduced MDA-MB-231 cell viability (Figure 4).

\section{SNCG Serves as a Target of miR-15a}

miRNAs usually play important roles in cellular functions by targeting critical downstream genes conserved across species. To explore the potential 
A)
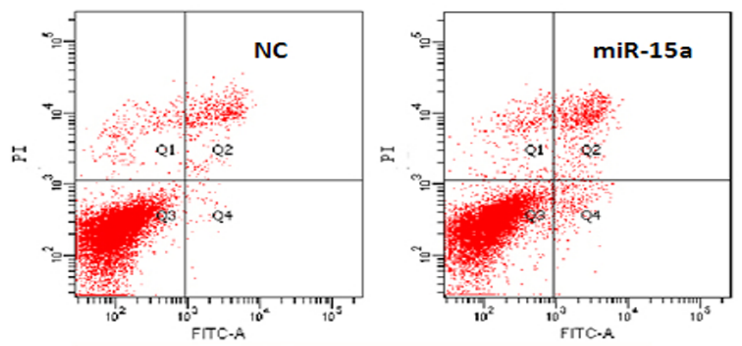

B)

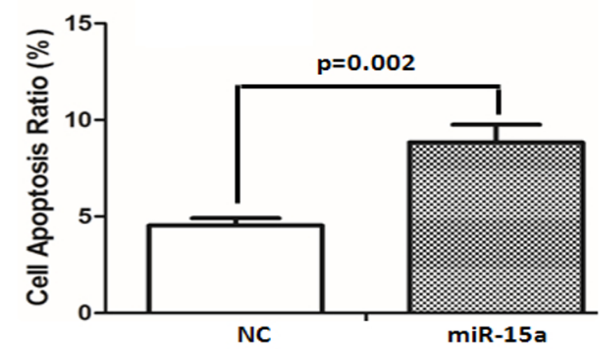

Figure 2. The High Expression of miR-15a Induces Apoptosis. A). Forty eight hours after MDA-MB-231 cells were transfected with $100 \mathrm{nM}$ of miR-15a mimics or NC. Cells were stained with Annexin V-FITC and PI and apoptosis were measured by flow cytometry. B). Representative histograms are shown. Transfection of MDA-MB-231 cells with miR-15a mimics caused statistically significant changes in the incidence of apoptosis in comparison to $\mathrm{NC}(\mathrm{n}=3$, mean $\pm \mathrm{SD})(p<0.05)$

A)
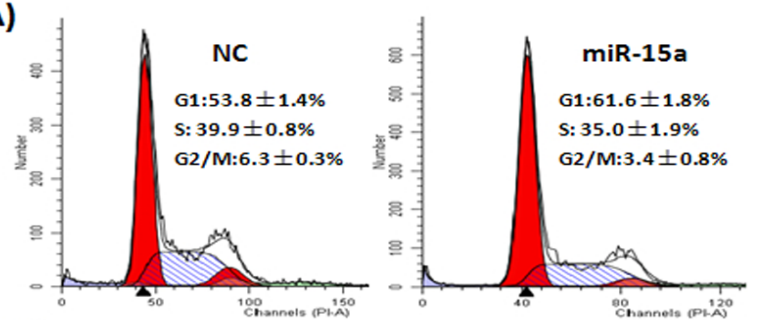

B)

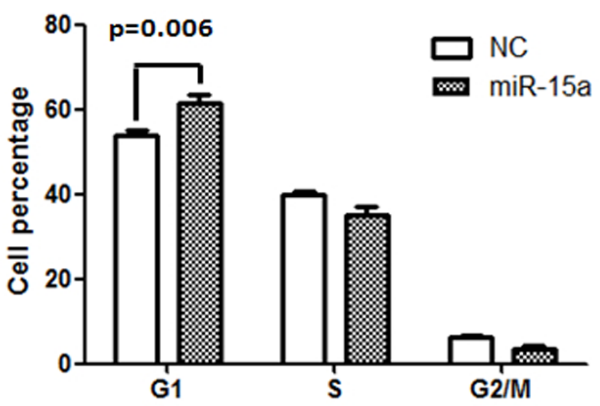

Figure 3. Cell Cycle was Analyzed by flow Cytometry. Forty Eight Hours After the MDA-MB-231 cells Were Transfected with the miR-15a mimics and NC at 100nM, Cell Cycle was Analyzed by flow Cytometry. A). Flow cytometry documents the G1 cell cycle arrest and inhibits $\mathrm{G} 1 / \mathrm{S}$ transition induced by upregulating miR15a compared with NC in MDA-MB-231 cells. B). The histogram showed the percentages of cells in G1, S and $\mathrm{G} 2 / \mathrm{M}$ phases $(\mathrm{n}=3$, mean \pm SD $)$

molecular mechanisms whereby miR-15a suppresses $\mathrm{BC}$ cells, we used two common computational methods to help identify miR-15a targets (Figure 5A, 5B). Bioinformatic analysis showed that SNCG genes' 3'UTR contains a target sequence of miR-15a that was completely complementary to the 2-8 nt of the miR-15a.

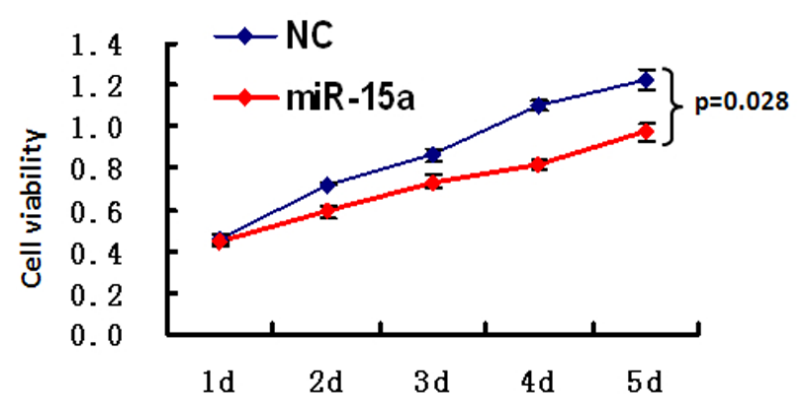

Figure 4. MiR-15a Suppresses Cell Viability. MDAMB-231 cells were transfected with miR-15a mimics and $\mathrm{NC}$, absorbance value of OD 450 was detected by CCK- 8 assay at $24 \mathrm{~h}$ intervals for 5 days $(\mathrm{n}=3$, mean $\pm \mathrm{SD})(p<0.05)$
A)

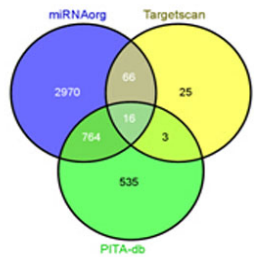

C)

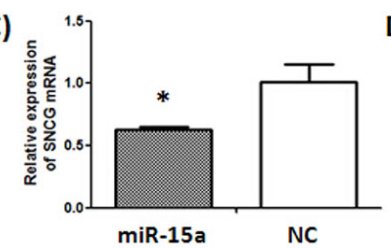

B)

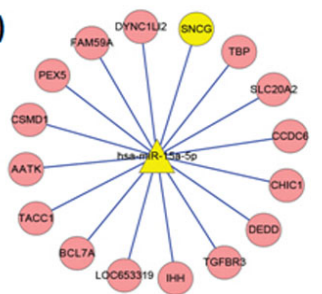

D)

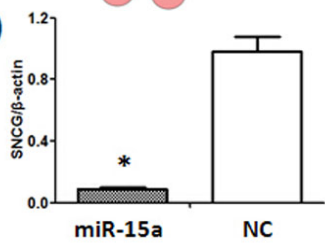

E)

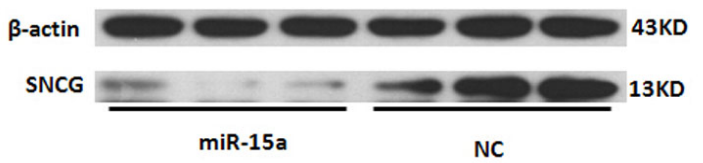

Figure 5. SNCG targeted miR-15a by Binding to its 3'- UTR. A). Venn diagram of miR-15a target genes prediction results from three databases (Targetscan, PITA and microRNAorg). B). There were 16 overlapping target genes predicted by three databases. C). The mRNA levels of SNCG was determined by qRT-PCR analyses after transfection with miR-15a mimics or NC in MDA-MB-231 cells. D, E). The protein of SNCG was determined by western blot analyses after transfection of miR-15a mimics or NC in MDA-MB-231 cells $(n=3$, mean $\pm \mathrm{SD}$ )

The mRNA level of SNCG analyzed by qRT-PCR was lower in the miR-15a mimics transfection group than in the $\mathrm{NC}$ transfection group $(p<0.05)$ (Figure $5 \mathrm{C}$ ). Western blot analysis of SNCG protein expression levels showed that SNCG levels in the miR-15a mimics transfection group were significantly lower than those in the $\mathrm{NC}$ transfection group $(p<0.05)$ (Figure 5D, 5E).

We constructed a luciferase reporter vector with the putative SNCG-3'UTR target site for miR-15a downstream of the luciferase gene (psiCHECK2-SNCG 3'UTR). A mutant version of psiCHECK2-SNCG 3'UTR with a 10 bp mutation within the seed region was also generated (psiCHECK2-SNCG 3'UTR Mutant) (Figure 6A). The luciferase reporter vector, in conjunction with miR-15a mimics or NC, was transfected into MDAMB-231 cells. As expected, a significant decrease in relative luciferase activity was noted when psiCHECK2- 
A)
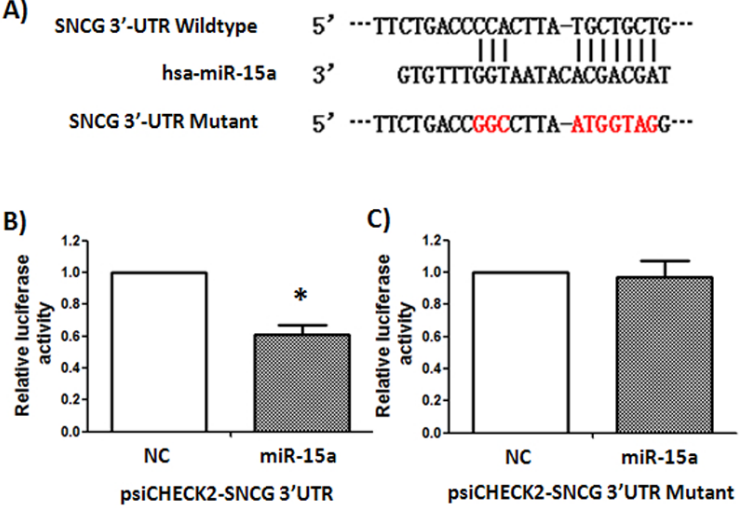

Figure 6. miR-15a Targets SNCG gene.A). The putative miR-15a binding site in the 3'UTR sequence of the SNCG gene. B, C). Luciferase activity assays of luciferase reporters with wild-type or mutant SNCG 3'UTR were performed after the Renilla luciferase activity $(n=3$, mean $\pm \mathrm{SD}$ )

SNCG 3'UTR was co-transfected with miR-15a mimics but not with NC (Figure 6B). However, the suppression was abolished by mutation of the 3'UTR miR-15a binding site (psiCHECK2-SNCG 3'UTR Mutant), which disrupts the interaction between miR-15a and the SNCG-3'UTR (Figure 6C).

\section{Discussion}

The occurrence, development, and metastasis of BC are closely associated with mutations in a variety of genes, alterations in cell signaling pathways, and neovascular dysplasia (Qin et al., 2012; Wei et al., 2012). MiRNAs act as "hackers" in the field of genetic research, as they regulate up to $30 \%$ of the protein- coding genes in humans. Numerous studies have demonstrated that miRNA abnormalities are closely related to the tumorigenesis of multiple types of tumors (Thorsen et al., 2012; Cortez et al., 2012; Huang et al., 2013). Besides, some miRNAs could be potential molecular biomarkers for predicting and monitoring resistance or sensitivity to chemotherapeutic drug of cancer patients (Chen et al., 2013; Yu et al., 2013).

In the present study, we showed that miR-15a was downregulated in $\mathrm{BC}$ tissues. We further showed that overexpressed miR-15a in BC cells induced G1 arrest, suppressed cell proliferation, and induced apoptosis. These data indicate that miR-15a may act as a tumor suppressor to inhibit cell proliferation by blocking the G1/S transition of $\mathrm{BC}$ cells. In other words, reduced miR-15a expression in $\mathrm{BC}$ cells and tissues may promote cell proliferation by activating the cell cycle.

MiR-15a regulates the apoptosis and proliferation of cells by functioning in the regulation of multiple intracellular signaling pathways. Luo et al. revealed that overexpression of miR-15a inhibited cellular growth, suppressed migration and arrested cells at the G1 phase, but did not promote cellular apoptosis (Luo et al., 2013). Cai et al. found that miR-15a downregulate CCND1 and induce apoptosis and cell cycle arrest in osteosarcoma (Cai et al., 2012). Regarding apoptosis, our results are not consistent with Luo's, potentially due to miR-15a having a different target gene. In this study, we utilized CCK-8 assays and flow cytometry analyses to determine the effects of miR-15a on MDA-MB-231 cells. The results of this study are consistent with the previously reported effects of miR-15a in other types of tumors.

SNCG is a small $13 \mathrm{KD}$ protein that is expressed in BC. SNCG predicts poor clinical outcome in BC (Guo et al., 2007; Wu et al., 2007). When overexpressed, SNCG stimulates growth of hormone-dependent BC cells both in vitro and in nude mice (Jiang et al., 2003; Jiang et al., 2004). Expression of SNCG in the mammary gland of transgenic mice induces a highly proliferative pregnancylike phenotype of mammary epithelial cells and gland hyperplasia (Liu et al., 2007). The contribution of SNCG to $\mathrm{BC}$ development and progression may be due to its chaperone activity in both estrogen (E2)-dependent and E2-independent pathways. SNCG participates in the heat shock protein 90 (Hsp90)-based multichaperone complex for steroid receptors and stimulates ER- $\alpha 66$ transcriptional activity but does not affect ER- $\beta$ signaling (Jiang et al., 2003; Jiang et al., 2004). The previous study demonstrated SNCG as a tumor specific chaperone, which can replace the chaperoning function of Hsp90 and protect and stimulate ER- $\alpha 36$-mediated MIES. Using a luciferase reporter gene system, the present study, confirmed for the first time that SNCG is a target gene of miR-15a. Our western blot results revealed that upregulation of miR-15a expression inhibits SNCG protein expression. Therefore, we argue that miR-15a affects MDA-MB-231 cell viability, proliferation, and apoptosis by regulating the expression levels of the SNCG protein.

In summary, miR-15a expression was downregulated in $\mathrm{BC}$ tissues and cells, and can negatively regulate SNCG protein expression. MiR-15a plays a vital role in inhibiting cell proliferation, mediating cell cycle arrest, and promoting apoptosis of MDA-MB-231 cells. Treatment strategies based on the upregulation of miR$15 \mathrm{a}$ expression or the low expression of the SNCG gene are very promising and may greatly benefit $\mathrm{BC}$ patients.

\section{Acknowledgements}

This work was supported by the National Basic Research Program of China (973 Program) (2012CB720600, 2012CB720605).

\section{References}

Ambros V (2001). MicroRNAs: tiny regulators with great potential. Cell, 107, 823-6.

Bartel DP (2004). MicroRNAs: Genomics, biogenesis, mechanism, and function. Cell, 116, 281-97.

Cai CK, Zhao GY, Tian LY, et al (2012). miR-15a and miR-16-1 downregulate CCND1 and induce apoptosis and cell cycle arrest in osteosarcoma. Oncol Rep, 28, 1764-70.

Chen C, Ridzon DA, Broomer AJ, et al (2005). Real-time quantification of microRNAs by stem-loop RT-PCR. Nucleic Acids Res, 33, 179.

Chen Q, Xia HW, Ge XJ, et al (2013). Serum miR-19a predicts resistance to FOLFOX chemotherapy in advanced colorectal cancer cases. Asian Pac J Cancer Prev, 14, 7421-6.

Cortez MA, Welsh JW, Calin GA (2012). Circulating microRNAs 
as noninvasive biomarkers in breast cancer. Recent Results Cancer Res, 195, 151-61.

Ferlay J, Shin HR, Bray F, et al (2010). Estimates of worldwide burden of cancer in 2008: GLOBOCAN 2008. Int J Cancer, 127, 2893-917.

Garzon R, Heaphy CE, Havelange V, et al (2009). MicroRNA $29 \mathrm{~b}$ functions in acute myeloid leukemia. Blood, 114, 5331-41.

Guo J, Shou C, Meng L, et al (2007). Neuronal protein synuclein gamma predicts poor clinical outcome in breast cancer. Int J Cancer, 121, 1296-305.

Harquail J, Benzina S, Robichaud GA (2012). MicroRNAs and breast cancer malignancy: an overview of miRNA-regulated cancer processes leading to metastasis. Cancer Epidemiol Biomarkers Prev, 11, 269-80.

Huang F, Liu C, Shi YH, et al (2013). MicroRNA-101 inhibits cell proliferation, invasion, and promotes apoptosis by regulating cyclooxygenase- 2 in Hela cervical carcinoma cells. Asian Pac J Cancer Prev, 14, 5915-20.

Jiang Y, Liu YE, Goldberg ID, et al (2004). A novel heat-shock protein-associated chaperone, stimulates ligand-dependent estrogen receptor $\alpha$ signaling and mammary tumorigenesis. Cancer Res, 64, 4539-46.

Jiang Y, Liu YE, Lu A, et al (2003). Stimulation of estrogen receptor signaling by gamma synuclein. Cancer Res, $\mathbf{6 3}$, 3899-903.

Lian J, Zhang X, Tian H, et al (2009). Altered microRNA expression in patients with non-obstructive zoospermia. Reprod Biol Endocrinol, 7, 13.

Liu YE, Pu W, Jiang Y, et al (2007). Chaperoning of estrogen receptor and induction of mammary gland proliferation by neuronal protein synuclein gamma. Oncogene, 26, 2115-25.

Luo Q, Li X, Li J, et al (2013). MiR-15a is underexpressed and inhibits the cell cycle by targeting CCNE1 in breast cancer. Int J Oncol, 43, 1212-8.

$\mathrm{Ng} \mathrm{SB}$, Yan J, Huang G, et al (2011). Dysregulated microRNAs affect pathways and targets of biologic relevance in nasaltype natural killer/T-cell lymphoma. Blood, 118, 4919-29.

Paik JH, Jang JY, Jeon YK, et al (2011). MicroRNA-146a Downregulates NFxB Activity via Targeting TRAF6 and Functions as a Tumor Suppressor Having Strong Prognostic Implications in NK/T Cell Lymphoma. Clin Cancer Res, 17, 4761-71.

Qin XJ, Ling BX (2012). Proteomic studies in breast cancer (Review). Oncol Lett, 3, 735-43.

Shenouda SK, Alahari SK (2009). MicroRNA function in cancer: oncogene or a tumor suppressor? Cancer Metastasis Rev, 28, 369-78.

Thorsen SB, Obad S, Jensen NF, et al (2012). The therapeutic potential of microRNAs in cancer. Cancer J, 18, 275-84.

Valencia-Sanchez MA, Liu J, Hannon GJ, et al (2006). Control of translation and mRNA degradation by miRNAs and siRNAs. Genes Dev, 20, 515-24.

Wang H, Tan G, Dong L, et al (2012). Circulating MiR-125b as a marker predicting chemoresistance in breast cancer. PLoS One, 7, 34210 .

Wei F, Xu J, Tang L, et al (2012). p27 (Kip1) V109G polymorphism and cancer risk: a systematic review and meta-analysis. Cancer Biother Radiopharm, 27, 665-71.

Wu K, Quan Z, Weng Z, et al (2007). Expression of neuronal protein synuclein gamma gene as a novel marker for breast cancer prognosis. Breast Cancer Res Treatment, 101, 259-67.

Yamanaka Y, Tagawa H, Takahashi N, et al (2009). Aberrant overexpression of microRNAs activate AKT signaling via down-regulation of tumor suppressors in natural killer-cell lymphoma/leukemia. Blood, 114, 3265-75.

Yu Q, Liu SL, Wang H, et al (2013). miR-126 Suppresses the proliferation of cervical cancer cells and alters cell sensitivity to the chemotherapeutic drug bleomycin. Asian Pac J Cancer Prev, 14, 6569-72. 